\section{PCa: Liegt der Grundstein in jungen Jahren?}

\section{Mehrere Faktoren im frühen Mannesalter können zur Entstehung eines Prostatakarzinoms ( $\mathrm{PCa}$ ) beitragen.}

S chon lange weiß man, dass etwa ein fortgeschrittenes Alter und eine positive Familienanamnese mit einem erhöhten Erkrankungsrisiko assoziiert sind. Australische Forscher haben nun nach weiteren Risikofaktoren gesucht.

Für die Fall-Kontroll-Studie verwendeten Visalini Nair-Shalliker und ihre Kollegen die Befunde der Daten- und Biobank CLEAR sowie die Ergebnisse einer Befragung. Die Datenbank enthält Informationen unter anderem zu allen Tumorarten bei Erwachsenen in diesem Bundesstaat, und zwar von mehr als 10.000 Menschen, davon etwa 8.500 Krebspatienten. Als Kontrollgruppe dienten männliche Lebenspartner von Erwachsenen dieser Gruppe ohne Krebserkrankung. Für die statistische Auswertung standen schließlich Daten von 1.181 Männern mit PCa und 875 Männern ohne Krebserkrankung zur Verfügung. Mithilfe der logistischen Regressionsanalyse wurde bestimmt, wie stark die Assoziation zwischen bestimmten Faktoren und der Entwicklung eines PCa war.

Fazit: Den stärksten Zusammenhang ermittelten die Forscher bei der positiven Familienanamnese. War der Vater an einem PCa erkrankt, lag die Wahrscheinlichkeit des Sohns, selbst daran zu erkranken, um $131 \%$ höher als ohne diesen Faktor (Odds Ratio [OR]: 2,31; 95\%-Konfidenzintervall zwischen 1,70 und 3,14; $\mathrm{p}$ $<0,001)$. Auch eine Prostatitis (OR: 2,30) und eine benigne Prostatahyperplasie (OR: 2,29) waren mit einer erhöhten Wahrscheinlichkeit assoziiert, genauso wie Übergewicht (OR: 1,24) und Adipositas (OR: 1,44). Schließlich hatten Männer, die sagten, bisher mehr als sieben
Geschlechtspartner gehabt zu haben, ein verdoppeltes Risiko, an Prostatakrebs zu erkranken (OR: 2,00). Und wer angab, vor der Diagnose mehr als fünf Orgasmen pro Monat gehabt zu haben, hatte mit einer $\mathrm{OR}=1,59 \mathrm{im}$ Vergleich zu Männern mit drei Orgasmen pro Monat ein um knapp 60 \% erhöhtes Risiko. Je früher der erste Geschlechtsverkehr war, umso höher war der Berechnung zufolge die Krebswahrscheinlichkeit (OR: 1,68, wenn der Zeitpunkt vor dem 17. Lebensjahr lag statt nach dem 22. Lebensjahr). Jedoch hätten andere Studien ein niedrigeres Risiko bei steigender Zahl der Geschlechtspartner ergeben, so die Epidemiologen. Keine Assoziationen konnten sie zwischen PCa-Risiko und Glatzenbildung, erektiler Funktion, Beschneidung, Vasektomie sowie Asthma und Diabetes entdecken.

Peter Leiner

Nair-Shalliker V et al. Adult body size, sexual history and adolescent sexual development, may predict risk of developing prostate cancer: Results from the New South Wales Lifestyle and Evaluation of Risk Study (CLEAR). Int J Cancer. 2016; doi: 10.1002/ijc.30471.

\title{
Neuer Ansatz gegen Tumorkachexie
}

\section{Viele Krebspatienten haben keinen Appetit und bauen deshalb körperlich ab. Das Hormon Ghrelin ist am Hungerstoffwechsel beteiligt. Ob Anamore- lin, ein Ghrelin-Rezeptoragonist, gegen Tumorkachexie helfen könnte, wurde in zwei parallel durchgeführten Phase-III-Studien untersucht.}

Bs eim Hungergefühl gesunder Menschen spielt der Stoffwechsel des Hormons Ghrelin eine wichtige Rolle. Deshalb könnte der neue Ghrelin-Rezeptoragonist Anamorelin womöglich onkologischen Patienten mit Kachexie helfen. Dies wurde in zwei parallel durchgeführten randomisierten, doppelblinden und placebokontrollierten Phase-III-Studien getestet.

An der ROMANA-1- und der ROMANA-2-Studie nahmen 93 Zentren in 19 Ländern teil. Alle Patienten litten unter einem inoperablen nicht kleinzelligen Lungenkarzinom (NSCLC) im Stadium III oder IV sowie unter Kachexie, das heißt ein Patient hatte binnen sechs Monaten mindestens $5 \%$ seines Körpergewichts verloren oder sein Body-Mass-In- dex (BMI) betrug weniger als $20 \mathrm{~kg} / \mathrm{m}^{2}$. Die Patienten wurden randomisiert, entweder täglich $100 \mathrm{mg}$ Anamorelin oral einzunehmen oder Placebo.

Zwischen Juli 2011 und Januar 2014 wurden 484 Patienten in die ROMANA1-Studie aufgenommen, 495 weitere $\mathrm{Pa}$ tienten zwischen Juli 2011 und Oktober 2013 in die ROMANA-2-Studie. Jeweils im Verhältnis 2:1 randomisiert erhielten sie entweder Anamorelin oder Placebo. Binnen 12 Wochen nahm die Magermasse des Körpers (Lean Body Mass, d.h. Körpermasse minus Speicherfett) bei $\mathrm{Pa}$ tienten im Anamorelin-Arm im Vergleich zur Kontrollgruppe signifikant zu: in der ROMANA-1-Studie im Mittel um $0,99 \mathrm{~kg}$ ( $\mathrm{p}<0,0001)$, in der ROMANA2-Studie um 0,65 kg ( $\mathrm{p}<0,0001)$. Ein stärkerer Handgriff wurde jedoch nicht festgestellt, auch zeigten sich keine Unterschiede im Hinblick auf behandlungsbedingte Nebenwirkungen vom Grad 3/4. Die häufigste dieser Nebenwirkungen war Hyperglykämie unter Anamorelin: bei 1 von 320 Patienten in der ROMANA-1- und bei 4 von 330 Patienten in der ROMANA-2-Studie.

Fazit: Der Ghrelin-Rezeptoragonist Anamorelin erhöhte das Körpergewicht von Patienten mit inoperablem NSCLC in zwei Phase-III-Studien im Vergleich zu Placebo signifikant. Der Handgriff der Patienten wurde jedoch nicht fester. $\mathrm{Da}$ es bisher kaum effektive Behandlungsmöglichkeiten für Kachexie gibt, könnte Anamorelin trotz dieses überschaubaren Erfolgs eine Option für Patienten mit krebsbedingter Anorexie oder Kachexie sein.

Christina Berndt

Temel JS et al. Anamorelin in patients with nonsmall-cell lung cancer and cachexia (ROMANA 1 and ROMANA 2): results from two randomised, double-blind, phase 3 trials. Lancet Oncol. 2016;17(4):519-31. 